\title{
A model of the heliocentric orbits of a stream of Earth-impacting interstellar meteoroids
}

\author{
W. J. Baggaley ${ }^{1,2}$ and L. Neslušan ${ }^{2,3}$ \\ 1 Department of Physics and Astronomy, University of Canterbury, Private Bag 4800, Christchurch, New Zealand \\ 2 Queen Mary College, Mile End Road, London E1 4NS, UK \\ 3 Astronomical Institute, Slovak Academy of Sciences, 05960 Tatranská Lomnica, Slovakia
}

Received 19 September 2001 / Accepted 16 November 2001

\begin{abstract}
We consider the influx of a collinear stream of interstellar particles (ISPs) into the Solar System for the case where non-gravitational forces are negligible (particle size $\gtrsim 20 \mu \mathrm{m}$ ) and model their Keplerian trajectories to yield the expected heliocentric orbits on impact with the Earth. The heliocentric orbital characteristics of such a stream of ISPs undergo cyclic seasonal variations allowing the determination of the stream inflow direction and far-Sun speed the orbit element signatures.
\end{abstract}

Key words. meteors, meteoroids - ISM: kinematics and dynamics

\section{Introduction}

Dust in the local interstellar medium (LIM) has a variety of sources: carbon-rich stars, red-giants, post AGB track stars, stars with debris disks, and supernovae. The proper solar motion in combination with the source ejection velocity of such collisionless dust grains will result in a stream of collinear interstellar particles (ISPs) flowing into the Solar System. The particles constituting such a collinear stream inflow to the Solar System will cross the Earth's orbit with particular trajectories. ISPs in the outer Solar System have been detected by spacecraft (Grün et al. 1993) and in the inner Solar System by ground-based techniques (e.g. Hawkes et al. 1999; Baggaley et al. 1999). The present work considers only large particles $(\gtrsim 20 \mu \mathrm{m})-$ those for which solar gravity dominates over radiation pressure, solar wind interaction, and Lorentz forces.

The ground-based determination of the orbits of Earth-impacting meteoroids in this size regime are available from multi-site facilities employing photographic, TV, image-intensifier video, and radar techniques. Such stereoscopic time-of-flight methods determine the atmospheric trajectories of meteoroids from the impact excitation and ionization generated by the ablating meteoroid.

The characteristics of the Earth-impacting orbits of an extra-solar collinear stream (having a width larger than the Solar System) of particles would be expected to undergo annual cyclic changes. Here we model the behaviour of the orbital elements of Earth-detected ISPs and show that their prominent seasonal signature is a valuable aid in

Send offprint requests to: L. Neslušan, e-mail: ne@ta3.sk seaking the presence of interstellar particle streams within the Solar System.

\section{The geometry and orbital elements of ISPs at $1 \mathrm{AU}$}

Since the gravity of the Sun deviates the trajectories of ISPs passing through the Solar System the direct recognition of the interstellar source of any Earth-impacting particles from their geocentric characteristics is not straightforward. The detection of a large number of hyperbolic orbits enables the fixing of the source direction from an accumulation of orbital incoming asymptotes (the heliocentric source directions) (Baggaley 1999).

All orbits in the solar gravitational field have to be located in the plane intersecting the Sun and since the meteor particles impact the Earth, their orbits have to intersect our planet at the instant of detection. The position of the Earth changes during the year resulting in a seasonal variation of orbital parameters of the detected particles. In this section, we give the explicit dependences of some of these parameters on the ecliptic longitude of the Earth.

ISPs entering the Solar System from a given heliocentric direction $\lambda, \beta$ (the upstream direction) and having a uniform monoenergetic far-Sun velocity $v_{\infty}$ can impact the Earth on two possible trajectories. For illustration, with $\beta$ negative, impacts can occur at the particle's ascending node (the particle's first nodal crossing) so the observed radiants are south of the ecliptic - or at the particle's descending node (the particle's second nodal 
crossing) impacting with radiants north of the ecliptic. These two possible dynamical solutions for Earth impact are here termed types 1 and 2 collisions respectively. Since ISPs follow heliocentric hyperbolic orbits, it is possible for only one node to exist (i.e. in this case type 1). Because of the different possible observational circumstances we distinguish the two types in the following.

Let us assume that the ISPs, on hyperbolic orbits, flow from a unique direction of interstellar space characterized by heliocentric ecliptical coordinates $\lambda$ and $\beta$ having direction cosines $s_{x}, s_{y}, s_{z}$. Let the ecliptic longitude, latitude, and heliocentric distance of the detection point be denoted by $\lambda_{\mathrm{E}}, \beta_{\mathrm{E}}$, and $r_{\mathrm{E}}$ respectively, the ecliptic rectangular coordinates of this point being $x_{\mathrm{E}}, y_{\mathrm{E}}, z_{\mathrm{E}}$. We identify the detection point with the Earth so $r_{\mathrm{E}}=1 \mathrm{AU}, \beta_{\mathrm{E}}=0^{\circ}$.

\subsection{The orbital inclination, $i$}

Utilizing the well known general equation of a plane in space, the orbital plane of the assumed interstellar particle has to satisfy the equations

$A s_{x}+B s_{y}+C s_{z}=0$

and

$A x_{\mathrm{E}}+B y_{\mathrm{E}}+C z_{\mathrm{E}}=0$,

where $A, B$, and $C$ are the constants of the orbital plane (the components of its normal vector). From relations (1) and (2), the constants $A$ and $C$ are related to $B$ by

$A=\frac{z_{\mathrm{E}} s_{y}-y_{\mathrm{E}} s_{z}}{x_{\mathrm{E}} s_{z}-z_{\mathrm{E}} s_{x}} B$

$C=\frac{y_{\mathrm{E}} s_{x}-x_{\mathrm{E}} s_{y}}{x_{\mathrm{E}} s_{z}-z_{\mathrm{E}} s_{x}} B$.

If the orbital plane is characterized by constants $(A, B$, $C, 0)$ (constant $D=0$ if the plane crosses the origin of coordinate system, i.e. the centre of the Sun in the present case), and the ecliptic is analogously characterized by constants $(0,0,1,0)$, then the cosine of the angle $\chi$ between the plane and the ecliptic can be expressed as

$$
\cos \chi=\frac{A .0+B .0+C .1+0.0}{\sqrt{\left(A^{2}+B^{2}+C^{2}+0^{2}\right)\left(0^{2}+0^{2}+1^{2}+0^{2}\right)}}
$$

or

$$
\begin{aligned}
\cos \chi= & \left(y_{\mathrm{E}} s_{x}-x_{\mathrm{E}} s_{y}\right)\left[\left(z_{\mathrm{E}} s_{y}-y_{\mathrm{E}} s_{z}\right)^{2}+\right. \\
& \left.+\left(x_{\mathrm{E}} s_{z}-z_{\mathrm{E}} s_{x}\right)^{2}+\left(y_{\mathrm{E}} s_{x}-x_{\mathrm{E}} s_{y}\right)^{2}\right]^{-1 / 2} .
\end{aligned}
$$

If a body in a prograde orbit has inclination $i=\chi$, then the orbit of a body in the same plane, but retrograde motion has inclination $180^{\circ}-\chi$. Hence if the particular ISP collides with the Earth in its first nodal point (type 1 geometry), then the inclination of its orbit is

$i=\chi$.
If it collides with the Earth in its second nodal point (type 2 geometry), then

$i=180^{\circ}-\chi$.

At any instant the Earth is impacted by ISPs in both prograde orbits and retrograde orbits.

\subsection{The longitude of ascending node, $\Omega$}

At the instant of a type 1 collision, if the ISP comes from a direction with $s_{z} \geq 0$, the longitude of the ISP's ascending node and the true anomaly $f_{\text {col. }}$ of the collision point are

$\Omega=\lambda_{\mathrm{E}}+180^{\circ} ; \quad f_{\text {col. }}=180^{\circ}-\omega$,

respectively. Conversely if $s_{z}<0$, then

$\Omega=\lambda_{\mathrm{E}} ; \quad f_{\text {col. }}=-\omega$,

with $\omega$ the argument of perihelion of the ISP's heliocentric orbit.

At the instant of a type 2 collision, if the ISP originally comes from the direction with $s_{z} \geq 0, \Omega$ and $f_{\text {col. }}$ are given by relation (10), and conversely if $s_{z}<0$ they are given by (9).

Taking into account the possible values of the true anomaly, the heliocentric distance of the collision point can be expressed (with $a$ and $e$ the semi-major axis and eccentricity of the ISP's heliocentric orbit) as

$r_{\mathrm{E}}=\frac{a\left(1-e^{2}\right)}{1+\sigma \cdot e \cdot \cos \omega}$,

where $\sigma=1$ for a type 1 collision and $s_{z}<0$ (or $s_{z} \geq 0$ for a type 2 collision); $\sigma=-1$ for a type 1 and $s_{z} \geq 0$ (or $s_{z}<0$ for a type 2 collision).

The reciprocal value of the semi-major axis then follows as

$1 / a=\frac{1-e^{2}}{r_{\mathrm{E}}(1+\sigma \cdot e \cdot \cos \omega)}$.

\subsection{The asymptote true anomaly, $f_{a}$}

If the true anomaly and orbital elements of the particle are known, then the rectangular coordinates of the particle follow using well known relations, while employing the heliocentric distance provides the directional cosines of the position of the particle. Knowing the true anomaly of the pre-perihelion asymptote $f_{\mathrm{a}}$ (the angle between the asymptote and line of apsides), then the relations determine the directional cosines of this asymptote, $s_{x}, s_{y}, s_{z}$. Specifically,

$s_{x}=\cos \left(\omega+f_{\mathrm{a}}\right) \cdot \cos \Omega-\sin \left(\omega+f_{\mathrm{a}}\right) \cdot \cos i \cdot \sin \Omega$,

$s_{y}=\cos \left(\omega+f_{\mathrm{a}}\right) \cdot \sin \Omega+\sin \left(\omega+f_{\mathrm{a}}\right) \cdot \cos i \cdot \cos \Omega$,

$s_{z}=\sin \left(\omega+f_{\mathrm{a}}\right) \cdot \sin i$.

Then explicit relations follow:

$\cos \left(\omega+f_{\mathrm{a}}\right)=s_{x} \cdot \cos \Omega+s_{y} \cdot \sin \Omega$ 
and

$\sin \left(\omega+f_{\mathrm{a}}\right)=\frac{s_{z}}{\sin i}$.

Relating the true anomaly and heliocentric distance, when the distance approaches infinity, $f_{\mathrm{a}}$ follows from

$1+e \cdot \cos f_{\mathrm{a}}=0$.

The angle $f_{\mathrm{a}}$ is in the range from $180^{\circ}$ to $270^{\circ}$, therefore

$\sin f_{\mathrm{a}}=-\sqrt{1-\cos ^{2} f_{\mathrm{a}}}=-\frac{\sqrt{e^{2}-1}}{e}$.

\subsection{The argument of perihelion, $\omega$}

Denoting

$C_{\omega+f}=\cos \left(\omega+f_{\mathrm{a}}\right)$ and $S_{\omega+f}=\sin \left(\omega+f_{\mathrm{a}}\right)$,

then from (18) and (19)

$\cos \omega=-\frac{1}{e} C_{\omega+f}-\frac{\sqrt{e^{2}-1}}{e} S_{\omega+f}$

$\sin \omega=\frac{\sqrt{e^{2}-1}}{e} C_{\omega+f}-\frac{1}{e} S_{\omega+f}$.

\subsection{The perihelion distance, $q$}

From the standard expression for the heliocentric speed in a Keplerian orbit, the speed outside the gravitational influence of the Sun can be written as

$v_{\infty}^{2}=-\frac{k^{2} M_{\odot}}{a}=k^{2} M_{\odot} \frac{e-1}{q}$,

where $k$ is the Gauss gravitational constant and $M_{\odot}$ is the mass of the Sun $\left(M_{\odot}=1\right.$ in units used).

\subsection{The eccentricity, e}

From (12) and (23), we obtain

$v_{\infty}^{2}=k^{2} M_{\odot} \frac{e^{2}-1}{r_{\mathrm{E}}(1+\sigma \cdot e \cdot \cos \omega)}$.

Defining further $U=r_{\mathrm{E}} \cdot v_{\infty}^{2} /\left(k^{2} M_{\odot}\right)$, then from $(21)$ and (24), we obtain an equation for the eccentricity the solution of which gives

$$
\begin{array}{r}
e=\left[1+\frac{1}{4}\left(\sqrt{U^{2} S_{\omega+f}^{2}+} 4 U\left(1-\sigma C_{\omega+f}\right)\right.\right. \\
\left.\left.-\sigma U S_{\omega+f}\right)^{2}\right]^{\frac{1}{2}} .
\end{array}
$$

\subsection{The distance of the pre-perihelion asymptote from the Sun, $b_{a}$}

A relevant quantity is the Sun's impact parameter, $b_{\mathrm{a}}$ : the distance of the pre-perihelion asymptote of the orbit of the ISP from the centre of the Sun (i.e. the distance of the point of asymptote nearest to the solar centre). From the appropriate geometry of the hyperbolic orbit, then

$\frac{b_{\mathrm{a}}}{|a|+q}=\left|\sin \left(360^{\circ}-f_{\mathrm{a}}\right)\right|$,

and using relations $(23),(18)$

$b_{\mathrm{a}}=k^{2} M_{\odot} \frac{\sqrt{e^{2}-1}}{v_{\infty}^{2}}$.

\section{The seasonal characteristics of ISP orbits}

As the Earth's heliocentric longitude $\lambda_{\mathrm{E}}$ changes, variations will occur in the collision geometry and therefore of the orbital elements of Earth intersecting ISPs.

The steps to calculate the characteristic orbital elements as a function of $\lambda_{\mathrm{E}}$ corresponding to date can be carried out as follows:

(1) Calculate the directional cosines $s_{x}, s_{x}$, and $s_{z}$ for assumed $\lambda, \beta$, and $v_{\infty}$.

(2) The ecliptic rectangular coordinates of the Earth, $x_{\mathrm{E}}$ and $y_{\mathrm{E}}\left(z_{\mathrm{E}}=0\right)$ can be calculated for a given date corresponding to the longitude of the Earth, $\lambda_{\mathrm{E}}$.

(3) The inclination $i$ of Earth intersecting orbits of the ISPs, when the Earth's ecliptic longitude is $\lambda_{\mathrm{E}}$, can now be calculated by relations (6) and, then, (7) or (8). The dependence of this orbital element on $\lambda_{\mathrm{E}}$ for several values $\beta$ is illustrated in Fig. 1. With the Earth's orbit circular, there is a simple relation between the ecliptic longitudes $\lambda$ and $\lambda_{\mathrm{E}}$. This fact permits the presentation of the corresponding behaviours for every $\lambda$, using the abscissa $D=\left(\lambda_{\mathrm{E}}-\lambda\right)$ : this variable is utilized in Fig. 1 and figures hereinafter.

Looking at relation (6), one can see that orbital inclination does not depend on velocity $v_{\infty}$. With respect to the invariance of inclination to $v_{\infty}$, an analysis of the orbital inclinations of ISPs appears to be the most efficient to reveal the presence of such particles.

(4) Relation (9) or (10) between $\Omega$ and $\lambda_{\mathrm{E}}$ is a priori valid for every meteor and therefore the seasonal variation of $\Omega$ is not useful as an identification of ISPs. However, it is necessary to evaluate $\Omega$ to obtain further set of quantities.

(5) Specifically, we need further to calculate the quantities $C_{\omega+f}$ and $S_{\omega+f}$ (see relation (20)) with help of relations (16) and (17).

(6) Subsequently, it is possible to calculate $e$ given by relation (25).

The seasonal variation of eccentricity for several combinations of $\beta$ and $v_{\infty}$ is presented in Fig. 2. Section 2 follows the convention $k \doteq 0.0172021 \mathrm{AU}^{3 / 2} M_{\odot}^{-1 / 2} \mathrm{day}^{-1}$ and $M_{\odot}=1$ so that velocities are $\mathrm{AU}_{\text {day }}{ }^{-1}$. In the figures $v_{\infty}$ is expressed in $\mathrm{km} \mathrm{s}^{-1}$. The two possible values of auxiliary quantity $\sigma$ in relation (25) mean that the colliding ISPs may move along two different orbits corresponding to the two dynamical solutions of the problem: types 1 and 2 collisions. In Fig. 2, the type 1 solutions are shown on the left plots (a, c, e), whilst the type 2 solutions are on right plots (b, d, f). 

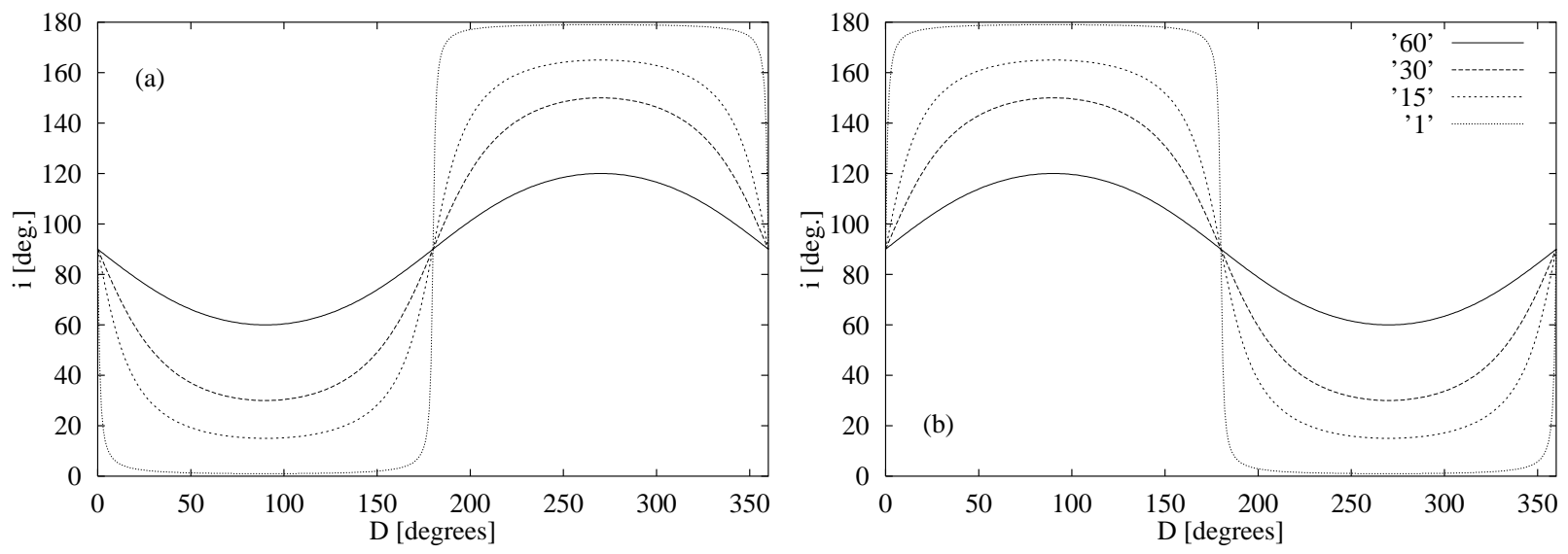

Fig. 1. The seasonal variation of orbital inclination, $i$, of an influx of hypothetical ISPs from the direction given by ecliptical coordinates $\lambda$ and $\beta$, with initial velocity $v_{\infty}$. The abscissa is the difference $D=\left(\lambda_{\mathrm{E}}-\lambda\right)$ relating $\lambda$ to the longitude of Earth, $\lambda_{\mathrm{E}}$. All behaviours are identical for $\beta$ and $-\beta$ and do not depend on the velocity $v_{\infty}$. There are four behaviours for values $|\beta|=1^{\circ}, 15^{\circ}, 30^{\circ}$, and $60^{\circ}$ in each plot. Plot a) gives the variation for the type 1 collision and plot $\mathbf{b}$ ) for the type 2 collision.


Fig. 2. The seasonal variation of eccentricity, $e$, of an influx of ISPs. The quantity $D$ in the abscissa is the same as in Fig. 1 . The first $\mathbf{a}), \mathbf{b}$ ), second $\mathbf{c}$ ), d), and third $\mathbf{e}), \mathbf{f}$ ) pairs of plots represent the seasonal variations for initial velocity, $v_{\infty}$, equal to 1,10 , and $20 \mathrm{~km} \mathrm{~s}^{-1}$, respectively. The left plots $\mathbf{a}$ ), c), e) illustrates the variation for the type 1 collision, the right plots $\mathbf{b}$ ), d), f) for the type 2 collision. Four curves in each plot correspond to the behaviours for the values of ecliptic lattitude $|\beta|=1^{\circ}$, $15^{\circ}, 30^{\circ}$, and $60^{\circ}$. 

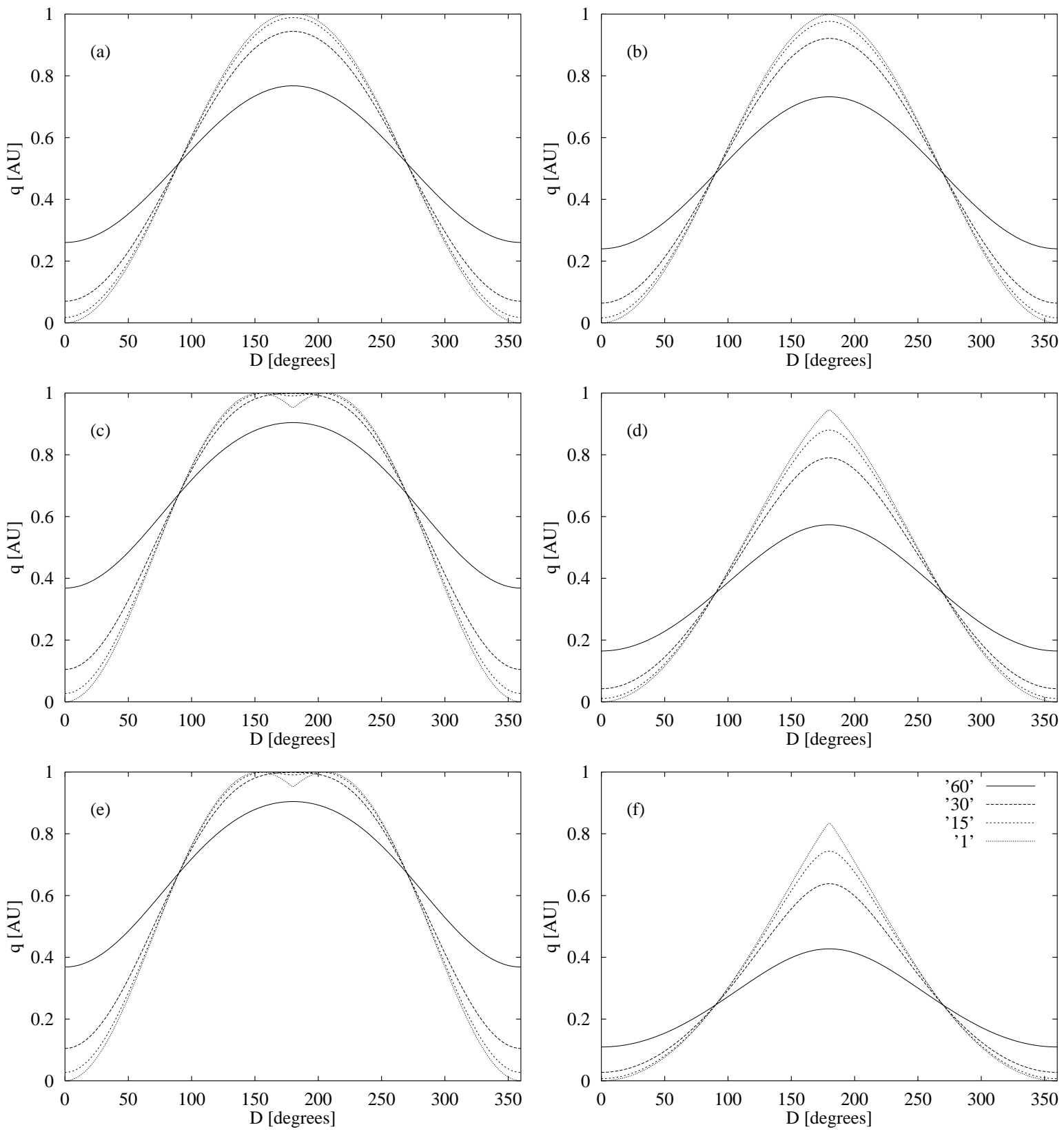

Fig. 3. The seasonal variation of perihelion distance, $q$, of an influx of ISPs. The quantity $D$ in the abscissa is the same as in Fig. 1. Four behaviours in every plot are given for the same values of ecliptic lattitude, $\beta$, as in Fig. 2. In analogy to Fig. 2, the plots from a) to f) correspond with the two types of collisions and with the three values of initial velocity.

(7) In the next step, we can compute the Sun's impact parameter, $b_{\mathrm{a}}$, by relation $(27)$.

(8) The seasonal variation of perihelion distance can be calculated by relation (23).

The resulting behaviour, for several $\beta$ and $v_{\infty}$ again, are plotted in Fig. 3. Comparing the corresponding plots in Figs. 2 and 3, very similar shapes of both behaviours for $e$ and $q$ are evident, a consequence of the linear relation between these elements (see relation (23)).

(9) Finally, the argument of perihelion, $\omega$, can be calculated, as a function of $\lambda_{\mathrm{E}}$, by relations (21) and (22).

The corresponding seasonal characteristics are given in Fig. 4 . In this case, we can see a special feature that $\omega$ is the same at $\left(\lambda_{\mathrm{E}}-\lambda\right)=90^{\circ}$ and $270^{\circ}$ for all values of $\beta$.
In contrast to the other parameters, the values of $\omega$ for $\beta$ and $-\beta$ are not the same, but shifted about $180^{\circ}$. These features are true for all values $v_{\infty}-$ in the type 1 as well as the type 2 collisions.

\section{The inverse problem: Determination of incoming direction and speed}

Looking at the variations in all four non-trivially variable elements $(i, e, q$, and $\omega)$, it is clear that the ecliptic longitude of the upstream direction the particles, $\lambda$, can be determined from all of the analysed elements. To determine $\beta$, the dependence of inclination on $\left(\lambda_{\mathrm{E}}-\lambda\right)$ has local extrema at values $\left(\lambda_{\mathrm{E}}-\lambda\right)=90^{\circ}$ and $270^{\circ}$ with $i=90^{\circ}$ at 
W. J. Baggaley and L. Neslušan: Interstellar-meteoroid-stream-orbit seasonal variation
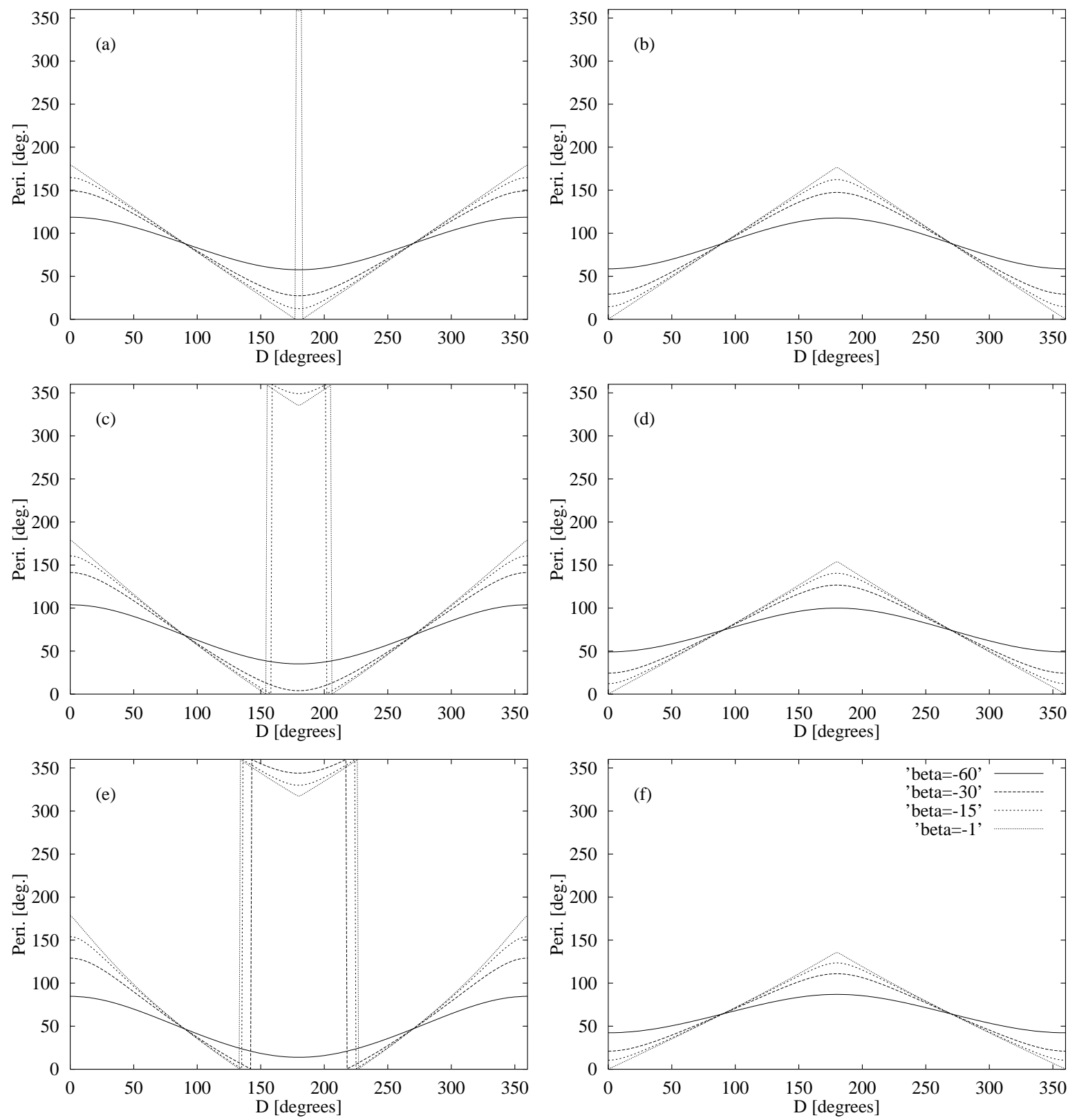

Fig. 4. The seasonal variation of argument of perihelion, Peri. $=\omega$, of an influx of ISPs. The quantity $D$ in the abscissa is the same as in Fig. 1. In analogy to Fig. 2, three pairs of plots are presented for the three values of initial velocity and the left and right plots correspond with the types 1 and 2 collisions, respectively. Four behaviours in each plot correspond to four values of ecliptic latitude $\beta=-60^{\circ},-30^{\circ},-15^{\circ},-1^{\circ}$ and while for $\beta \geq 0^{\circ}$ the corresponding behaviours are shifted about $180^{\circ}$ $\left[\omega(\beta)=\omega(-\beta)-180^{\circ}\right]$.

$\left(\lambda_{\mathrm{E}}-\lambda\right)=0^{\circ}$ and $180^{\circ}$. All variations of inclination (for every $\beta$ ) are symmetric with respect to these two points. A symmetry with respect to vertical axes crossing the abscissa in $0^{\circ}$ and $180^{\circ}$ exists in the seasonal variations of $e, q$, and $\omega$. The patterns of these three elements have moreover their local extrema at $\left(\lambda_{\mathrm{E}}-\lambda\right)=0^{\circ}$ and $180^{\circ}$.

The individual values in the local extrema of $e, q$, and $\omega$ determine the far-Sun velocity, $v_{\infty}$.

Any discrete astronomical source of ISPs would be expected to produce particles having a distribution in speed: such a dispersion would yield a dispersion in orbital elements (but not in $i$ ).
The sign of the ecliptic lattitude, $\beta$, can be, in principle, determined only from the behaviour of $\omega$ while the absolute value of $\beta$ can most simply be determined from the seasonal behaviour of inclination.

For heliocentric source directions close to the ecliptic poles the seasonal modulation in $i, e, q, \omega$ will be small: for $\beta=+90^{\circ}$ or $-90^{\circ}$ then $i=90^{\circ}$ and $v_{\infty}$ can be obtained from the (time invariant) values of $e, q$, and $\omega$.

\section{Discussion}

The time variations in the heliocentric orbital elements of a stream of ISPs as registered at the Earth undergo seasonal modulations. The characteristics allow the fixing 
of the heliocentric source direction and far-Sun speed. In contrast to the case of interplanetary closed orbit meteoroid streams (originating from short-period and Halley type comets) the behaviour for ISPs is quite different all year round observability and cyclic changes in the observed orbital elements. This behaviour is described by Keplerian dynamics where interstellar collisions, solar non-gravitational forces, and planetary scattering are negligible.

A stream of ISPs in the size regime $\gtrsim 20 \mu \mathrm{m}$ from a discrete source would be expected to undergo negligible interaction with the ambient interstellar grains and so retain an essentially collinear property for source distances $\$ 1$ kpc (Grün \& Landgraf 2000).

Solar radiation effects - pressure due to direct momentum transfer and Poynting-Robertson energy loss will be minor for grains of sizes $\gtrsim 20 \mu \mathrm{m}$ with single trajectories (e.g. Gustafson 1994). ISPs will acquire an electrical charge so that for small grains (size $\lesssim 1 \mu \mathrm{m}$ ) it is necessary to include interactions with the solar wind plasma and deviations produced by the ambient magnetic field within the heliopause (e.g. Grogan et al. 1996). No effects of the close approach of ISPs to the major planets have been considered. For out of ecliptic sources entry into the gravitational sphere of influence of planets is only relevant for type 2 geometry. We note that for the case of $v_{\infty}=20 \mathrm{~km} \mathrm{~s}^{-1}$ then for a trajectory deviation of $\sim 1^{\circ}$ the Jupiter approach distance required is $\sim 0.03 \mathrm{AU}$.

Acknowledgements. This work was carried out with support from the New Zealand Marsden Fund (UoC-85) (W.J.B.) and L.N. acknowledges the support by VEGA, the Slovak Grant Agency for Science (grant No. 1023).

\section{References}

Baggaley, W. J. 1999, in Meteoroids 1998, ed. W. J. Baggaley, \& V. Porubčan, Astron. Inst. Slovak Acad. Sci., Bratislava, 265

Baggaley, W. J., Taylor, A. D., \& Steel, D. I. 1993, in Meteoroids and Their Parent Bodies, ed. J. Štohl, \& I. P. Williams, Astron. Inst. Slovak Acad. Sci., Bratislava, 53

Baggaley, W. J., Taylor, A. D., \& Steel, D. I. 1994, Planet. Space Sci., 42, 135

Grogan, K., Dermott, S. F., \& Gustafson, B. A. S. 1996, ApJ, 472,812

Grün, E., Zook, H. A., Baguhl, M., et al. 1993, Nature, 362, 428

Grün, E., \& Landgraf, M. 2000, J. Geophys. Res., 105, 10291

Gustafson, B. A. S. 1994, Ann. Rev. Earth Planet. Sci., 22, 553

Hawkes, R., Close, T., \& Woodworth, S. 1999, in Meteoroids 1998, ed. W. J. Baggaley, \& V. Porubčan, Astron. Inst. Slovak Acad. Sci., Bratislava, 257 